\title{
Cytotoxic Lesion of the Corpus Callosum in a patient with Aphasic Status Epilepticus
}

DOI: https://doi.org/10.17981/10.17981/JACN.1.1.2020.15

\author{
Juan Ignacio Castiglione ${ }^{1}$, Mario Emiliano Ricciardi ${ }^{1}$ \& Catalina Bensi $^{1}$
}

\footnotetext{
${ }^{1}$ Neurology department, Fleni (Buenos Aires, Argentina)
}

Correspondence:

Juan Ignacio Castiglione

Neurology department, Fleni (Buenos Aires, Argentina) E mail:

jicastiglione@fleni.org.ar
A 47-year-old man with a history of aphasic seizures presented to the emergency room with a 12-hour global aphasia. Upon admission, brain MRI did not reveal acute lesions, and EEG showed sharp waves in the left frontal-temporal region. An Aphasic Status Epilepticus was diagnosed and antiepileptic treatment was initiated with adequate response.

A week after the episode, a new brain MRI showed a highsignal ovoid lesion on T2-weighted and FLAIR sequences in the central part of the splenium of the corpus callosum (Figure 1). On Diffusion-Weighted Images (DWI) the lesion was hyperintense with decreased Apparent Diffusion Coefficient (ADC) values, indicating restricted diffusion consistent with a Cytotoxic Lesion of the Corpus Callosum (CLOCC) (Takayama, Kobayashi, Sugishita \& Mihara, 2000; Conti et al., 2007). Follow-up MRI one month later showed complete image resolution (Figure 2).

CLOCCs are secondary lesions associated with various entities in which high levels of cytokines and extracellular glutamate cause intracellular edema and reduced diffusion, a condition called cytotoxic edema, which affects vulnerable brain regions such as the splenium of the corpus callosum (Starkey, Kobayashi, Numaguchi \& Moritani, 2017; Tetsuka, 2019).

In epileptic patients, CLOCCs may be due to the effect of seizures, especially prolonged ones, as well as antiepileptic treatment itself (Prilipko, Delavelle, Lazeyras \& Seeck, 2005; Polster, Hoppe \& Ebner, 2001). CLOCCs are rare radiological findings and must be recognized to avoid misdiagnosis. 

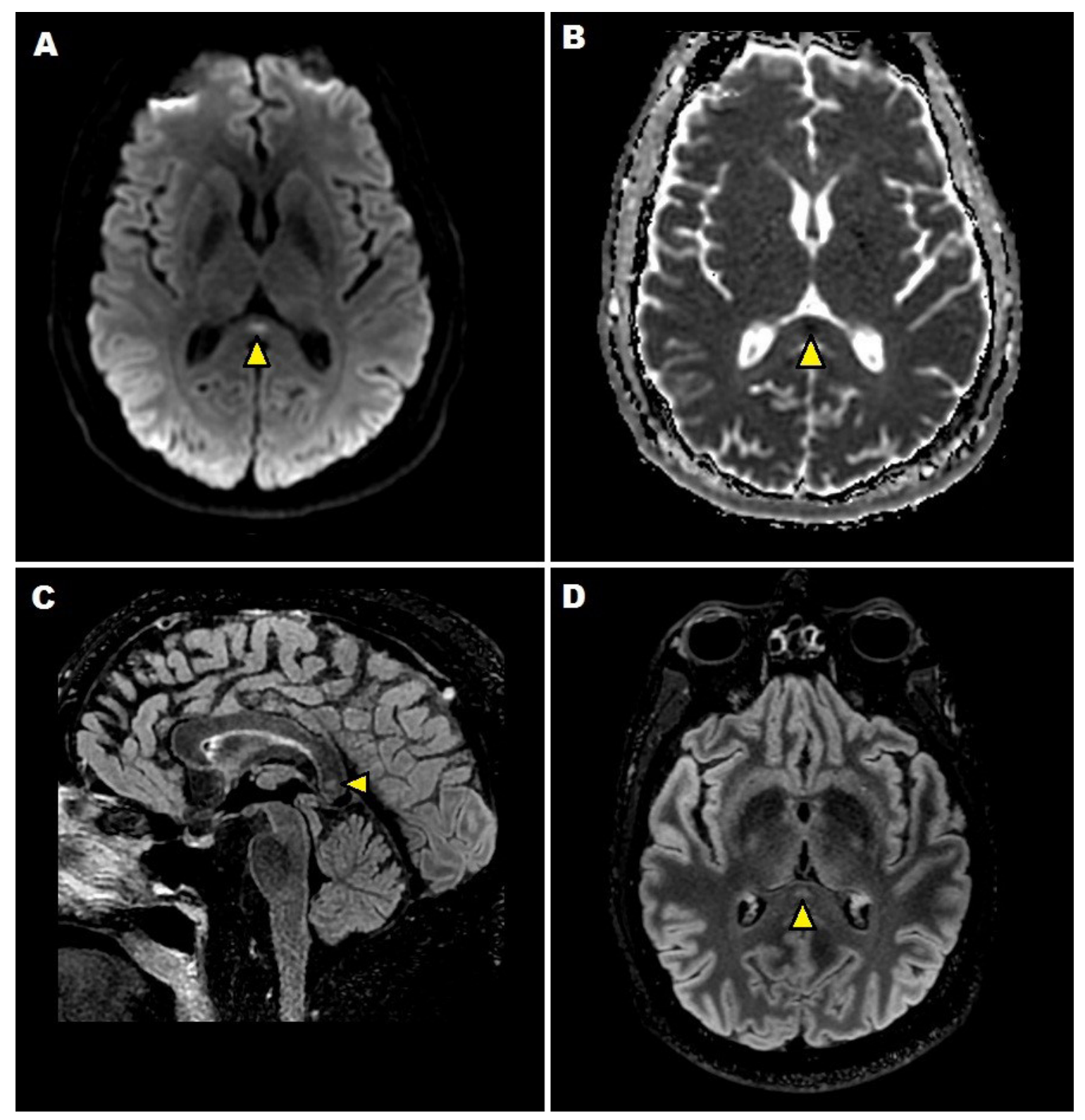

Figure 1. A-B. Axial DWI MRI image (A), and ADC map (B) show an ovoid focal lesion in the splenium. C-D. Sagital (C) and axial (D) FLAIR images revealed a slight hyperintense signal at the same location. Source: Authors.
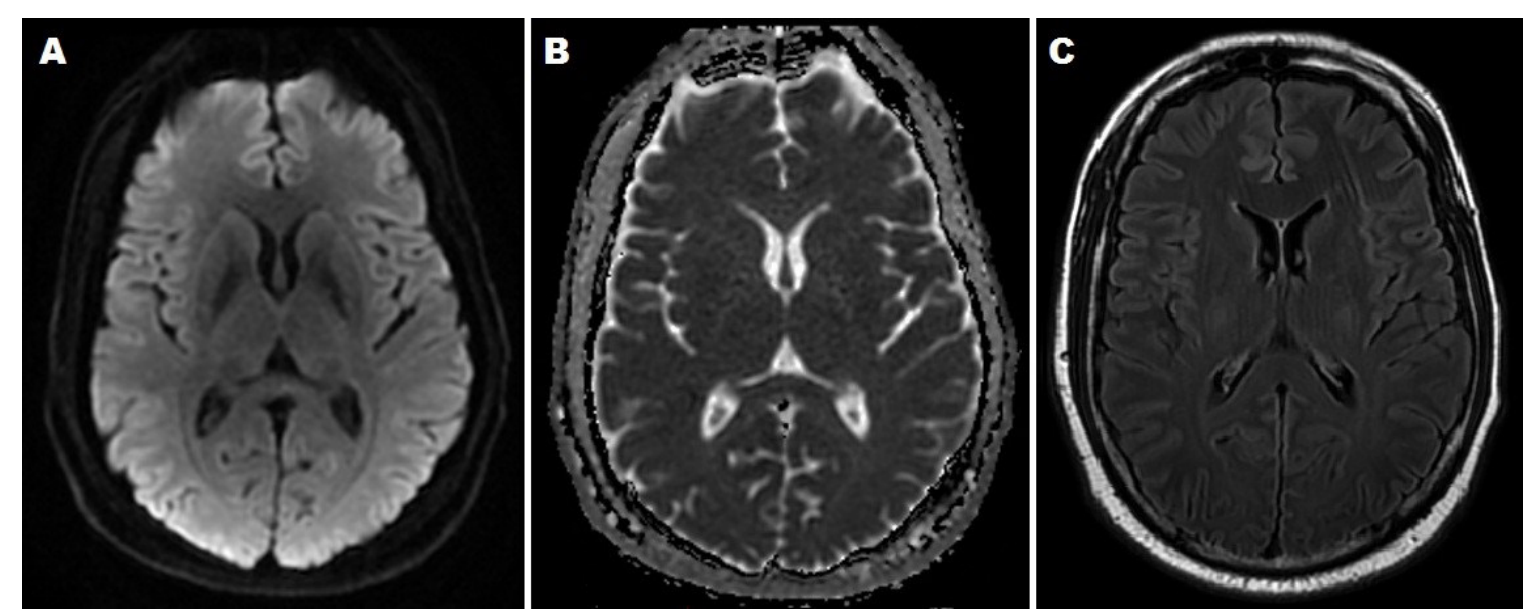

Figure 2. A-C. Follow-up MRI one month later shows complete resolution of the lesion in both DWI (A), ADC map (B) and FLAIR (C) images.

Source: Authors. 


\section{REFERENCES}

Conti, M., Salis, A., Urigo, C., Canalis, L., Frau, S. \& Canalis, G. C. (2007). Transient focal lesion in the splenium of the corpus callosum: MR imaging with an attempt to clinical-physiopathological explanation and review of the literature. La Radiología Médica, 112(6), 921-935. http://dx.doi.org/10.1007/s11547-0070197-9

Prilipko, O., Delavelle, J., Lazeyras, F. \& Seeck, M. (2005). Reversible cytotoxic edema in the splenium of the corpus callosum related to antiepileptic treatment: report of two cases and literature review. Epilepsia, 46(10), 1633-1636. http://dx.doi. org/10.1111/j.1528-1167.2005.00256.x

Polster, T., Hoppe, M. \& Ebner, A. (2001). Transient lesion in the splenium of the corpus callosum: three further cases in epileptic patients and a pathophysiological hypothesis. Journal of Neurology Neurosurgery Psychiatry, 70(4), 459-463. http://dx.doi.org/10.1136/jnnp.70.4.459
Starkey, J., Kobayashi, N., Numaguchi, Y. \& Moritani, T. (2017). Cytotoxic Lesions of the Corpus Callosum That Show Restricted Diffusion: Mechanisms, Causes, and Manifestations. Radiographics, 37(2), 562-576. http:// dx.doi.org/10.1148/rg.2017160085

Takayama, Hm., Kobayashi, M., Sugishita, M. \& Mihara, B. (2000). Diffusion-weighted imaging demonstrates transient cytotoxic edema involving the corpus callosum in a patient with diffuse brain injury. Clinical Neurology and Neurosurgery, 102(3), 135139. http://dx.doi.org/10.1016/s03038467(00)00079-2

Tetsuka, S. (2019). Reversible lesion in the splenium of the corpus callosum. Brain and behavior, 9(11), 1-10. https://doi.org/10.1002/brb3.1440

Juan Ignacio Castiglione: Medical (University of Buenos Aires, Argentina).

Mario Emiliano Ricciardi: Neurology resident Medical Fleni (Buenos Aires, Argentina).

Catalina Bensi: Medical (University of Buenos Aires, Argentina). 